\title{
Microbiote intestinal et lipides : impact sur la santé humaine
}

Philippe GÉRARD

INRA,

UMR1319 Micalis,

Equipe AMIPEM,

Domaine de Vilvert,

F-78350 Jouy-en-Josas,

France;

AgroParisTech,

UMR Micalis,

F-78350 Jouy-en-Josas,

France

$<$ philippe.gerard@jouy.inra.fr>

Article reçu le 3 mai 2012

Accepté le 10 mai 2012

Le microbiote intestinal, I'ensemble des bactéries qui peuplent notre tube digestif, est en contact direct avec les cellules de notre intestin. À plus d'un titre, cette association peut être considérée comme mutualiste et les micro-organismes qui nous colonisent sont responsables de nombreuses fonctions essentielles au maintien de notre santé, au point que l'on peut considérer ce microbiote comme un organe supplémentaire de notre organisme. Le dialogue qui s'établit entre ce microbiote et notre intestin peut par ailleurs être modulé par différents facteurs et en particulier I'alimentation. Dans cet article, nous nous intéresserons en particulier aux relations entre le microbiote intestinal et les lipides, ainsi qu'à leurs conséquences physiologiques pour I'hôte.

\section{Le microbiote intestinal}

Le nouveau-né, stérile in utero, se retrouve à la naissance en contact avec des bactéries qui vont rapidement

\begin{abstract}
Gut microbiota and lipids: impact on human health
The human intestine harbours a complex and diverse bacterial community called the gut microbiota. This microbiota, stable during the lifetime, is specific of each individual despite the existence of a phylogenetic core shared by the majority of adults. The influence of the gut microbiota on host's physiology has been largely studied using germfree animals and recently it has been proposed that the gut microbiota affects nutrient acquisition, energy regulation and fat storage. Indeed, germfree animals are resistant to diet induced obesity and display low levels of blood and liver lipids.

In humans, several grams of lipids reach the colon each day. These lipids have an impact on the gut microbiota composition characterized by an increase of the Firmicutes/ Bacteroides ratio. Concurrently, the gut microbiota is able to convert lipids, including fatty acids or cholesterol, leading to the production of metabolites with potential health effects.
\end{abstract}

Key words: gut microbiota, high-fat diet, germ-free animals, lipids, obesity

coloniser son tube digestif. Le microbiote fécal maternel constitue la source principale de bactéries colonisatrices mais le microbiote vaginal participe également à la colonisation (Palmer et al., 2007). D'autres bactéries, provenant de la nourriture, de l'environnement ou du contact avec les parents, entrent ensuite en contact avec le nouveau-né et participent à la complexification progressive du microbiote de l'enfant. Ce n'est que vers l'âge de 2, voire 4 ans qu'un microbiote stable, proche de celui de l'adulte, sera définitivement installé.

Chez l'adulte, si la densité n'est que de quelques centaines de bactéries par gramme de contenu dans l'estomac, elle augmente à mesure que l'on avance dans l'intestin pour atteindre son maximum dans le côlon distal avec $10^{11}$ bactéries par gramme. Au total, ce sont donc plus de $10^{14}$ bactéries qui colonisent notre tube digestif, c'est-à-dire dix fois plus que le nombre de cellules eucaryotes constituant le corps humain. Ces bactéries, environ mille espèces différentes, représentent approximativement $1 \mathrm{~kg}$ de notre masse corporelle. À cela s'ajoute la présence d'eucaryotes unicellulaires tels que des levures ou des protozoaires dont l'importance, en termes de niveau de population et de fonction, est encore mal connue.

Jusqu'aux années 1980, la caractérisation du microbiote intestinal était exclusivement réalisée à l'aide de techniques de culture, ne prenant en compte que $30 \%$ environ des microorganismes présents. Des outils moléculaires ont depuis été développés et ont permis de montrer que la plus grande partie des espèces dominantes observées dans le microbiote fécal d'un individu lui sont propres, même si, un petit nombre d'espèces (quelques dizaines) pourraient constituer un cœur phylogénétique partagé par la plupart des individus (Tap et al., 2009). De même, l'analyse de sa composition en taxa (genres bactériens et/ou grands groupes phylogénétiques) fait ressortir l'existence de composantes récurrentes, retrouvées chez tous les individus. Trois 
phyla bactériens, Firmicutes, Bacteroidetes et Actinobacteria, rassemblent la plus grande part des bactéries intestinales dominantes. Le phylum des Firmicutes (bactéries à Gram positif) est en général le phylum le plus représenté, réunissant couramment plus de la moitié des bactéries présentes. Les Bacteroidetes sont également toujours présents et partagent la dominance avec les Firmicutes puisqu'ils représentent 10 à $30 \%$ des bactéries totales. Le phylum Actinobacteria est moins systématiquement détecté en dominance et représente en général moins de $10 \%$ des bactéries totales. Les autres groupes bactériens sont plus rarement observés dans le microbiote dominant. Ainsi les lactobacilles et streptocoques représentent en général moins de $2 \%$ et les entérobactéries moins de $1 \%$ des bactéries totales. Par ailleurs, des espèces appartenant au domaine Archae sont présentes dans le microbiote fécal dominant de la moitié environ des adultes dans les pays occidentaux.

Si la composition du microbiote intestinal varie d'un individu à l'autre, le profil des espèces dominantes semble remarquablement stable dans le temps pour un individu donné sur des échelles de temps allant de quelques jours à plusieurs années. Néanmoins, un changement de régime alimentaire est susceptible de modifier, au moins partiellement, l'équilibre de l'écosystème intestinal. Cependant, même un stress majeur tel qu'un traitement antibiotique est suivi d'un retour au profil initial d'espèces dominantes au bout d'environ un mois (De La Cochetiere et al., 2005). Cette aptitude à revenir à l'équilibre initial après un stress, la résilience, suggère une adaptation très forte du microbiote à l'intestin voire à l'hôte qui I’héberge.

\section{Les fonctions du microbiote intestinal}

Le microbiote intestinal exerce de nombreuses fonctions physiologiques dont les répercussions pour I'hôte sont, pour la plupart, bénéfiques. De plus, si la composition phylogénétique du microbiote intestinal varie d'un individu à l'autre, il semble que les fonctions qu'il exprime sont pour la plupart partagées. Parmi les grandes fonctions du microbiote, la fermentation des substrats disponibles au niveau du côlon, le rôle de barrière à la colonisation par les micro-organismes pathogènes, la synthèse de vitamines, le développement et la maturation du système immunitaire intestinal et les interactions avec les cellules épithéliales participent au maintien de la santé de I'hôte. La biotransformation par le microbiote des différents substrats parvenant au côlon implique, de plus, l'existence de nombreuses activités métaboliques des micro-organismes en présence. Ces activités microbiennes sont importantes pour l'hôte puisque les métabolites formés sont, pour la plupart, absorbés et utilisés dans l'organisme et ont ainsi des répercussions sur la nutrition et la santé.

Une des façons de mettre en évidence l'influence du microbiote intestinal sur la physiologie de l'hôte consiste à étudier des animaux dépourvus de microbiote intestinal (ces animaux sont alors dits sans germes ou axéniques). Des élevages d'animaux axéniques existent depuis plus d'un demi-siècle et nécessitent des enceintes stériles appelées isolateurs. L'élevage débute par la collecte et l'introduction dans ces isolateurs d'utérus de femelles gravides. La descendance, axénique, peut ensuite être élevée en isolateurs sur plusieurs générations. Les animaux sans germes présentent ainsi un certain nombre d'anomalies physiologiques qui peuvent être corrigées en quelques semaines par l'inoculation d'un microbiote complexe, et parfois, d'une seule espèce bactérienne. L'observation de ces animaux a ainsi démontré l'importance du microbiote sur le développement et la maturation du système immunitaire de l'hôte, au niveau intestinal mais également au niveau périphérique (Smith et al., 2007). Plus récemment, le rôle joué par le microbiote dans le métabolisme lipidique de l'hôte et son implication dans le développement de l'obésité ont été révélés.

\section{Microbiote intestinal et métabolisme de I'hôte}

Dès 1983, Wostmann et al avaient observé que des rongeurs axéniques avaient besoin de $30 \%$ de calories supplémentaires pour maintenir leur masse corporelle par rapport à des animaux conventionnels (Wostmann et al., 1983). Cependant, les mécanismes permettant d'expliquer cette observation restèrent inconnus jusqu'à des travaux récents menés par l'équipe de Jeffrey Gordon (St-Louis, USA). Leur première étude a ainsi montré que la quantité de masse grasse présente chez des souris conventionnelles était $42 \%$ supérieure à celles de souris sans germes du même âge et de la même souche (Backhed et al., 2004). La colonisation de ces souris axéniques avec un microbiote intestinal aboutit à une augmentation de $60 \%$ de la masse grasse en deux semaines malgré une réduction de la prise alimentaire de $30 \%$ (Backhed et al., 2004). Un des mécanismes invoqués pour expliquer ce phénomène est l'inhibition de l'expression de la protéine Fiaf (Fasting-induced adipocyte factor) dans les cellules épithéliales de l'iléum suite à l'inoculation d'un microbiote. Fiaf étant un inhibiteur circulant de la lipoprotéine lipase, la présence du microbiote aboutit donc à une activité lipoprotéine lipase plus élevée et ainsi à une augmentation du stockage de triglycérides dans les adipocytes. La même équipe a ensuite montré que les souris sans germes restent minces en réponse à un régime riche en graisses (Backhed et al., 2007). Lors d'une étude récente, nous avons essayé de comprendre ce phénomène en comparant des souris adultes mâles C57BL/6] axéniques et conventionnelles, nourries pendant 3 mois avec un aliment hyperlipidique (60\% des calories d'origine lipidique) distribué à volonté (Rabot et al., 2010). Comme attendu, les souris axéniques prennent moins de poids ( 3 fois moins) que les souris conventionnelles, cette différence pouvant ici s'expliquer partiellement par une moindre consommation alimentaire $(-15 \%)$ et une excrétion fécale de lipides supérieure (+ $25 \%$ ). Le régime hyperlipidique a des conséquences différentes sur I'homéostasie lipidique des souris selon leur statut bactérien. A jeun, les souris axéniques ont moins d'acides gras libres, de triglycérides et de cholestérol (total et HDL) dans le sang, et moins de triglycérides mais plus de cholestérol dans le foie. Ces particularités s'accompagnent d'une augmentation coordonnée dans le tissu hépatique (i) de l'expression de gènes codant des enzymes impliquées dans la biosynthèse des stéroïdes (e.g. HMG-CoA synthétase et réductase), (ii) de la concentration nucléaire du facteur de transcription SREBP2 qui contrôle la synthèse de ces enzymes. Les souris axéniques ont aussi une meilleure sensibilité à I'insuline que les souris conventionnelles, comme I'indiquent (i) une glycémie et une insulinémie (à jeun ou non) inférieures, (ii) une plus grande tolérance au glucose et (iii) une augmentation de la forme 
phosphorylée de la protéine kinase B (Akt) dans le tissu adipeux viscéral (Rabot et al., 2010). Ces résultats démontrent l'importance du microbiote intestinal dans la régulation du métabolisme des glucides et des lipides chez l'hôte. L'intestin étant en contact direct avec le microbiote, on peut supposer que ces effets sont, au moins partiellement, dues à des modifications du métabolisme lipidique intestinal. Très récemment, il a ainsi été montré que l'expression basale des gènes clefs de l'absorption lipidique (CD36, L-FABP, MTP) est modifiée au niveau de l'intestin de souris axéniques en comparaison à des souris conventionnelles (Larsson et al., 2011).

\section{Effets d'un régime riche en lipides sur le microbiote intestinal}

Si les études portant sur les effets de polysaccharides non digestibles sur la composition du microbiote intestinal existent depuis des décennies, l'influence des lipides sur ce microbiote n'a été étudiée que récemment, suite aux travaux liant microbiote intestinal et obésité. Ainsi, Turnbaugh et al. ont suivi I'évolution du microbiote intestinal de souris soumises à un régime riche en lipides. Ce régime aboutit à une chute de la diversité du microbiote, due en partie à l'émergence d'un groupe bactérien membre des Firmicutes : Erysipelotrichi (Turnbaugh et al., 2008). Le retour à un régime hypocalorique conduit à une perte de poids des souris et à une forte diminution de la proportion d'Erysipelotrichi. Néanmoins, une autre étude, si elle a confirmé l'effet d'un régime hyperlipidique sur la composition du microbiote intestinal, suggère que cette modification du microbiote est indépendante de l'obésité (Hildebrandt et al., 2009). Par ailleurs, il a été montré que la modification de la composition du microbiote intestinal par un régime hyperlipidique s'accompagne d'une augmentation des taux circulants de lipopolysaccharides (Ips), composants de la paroi des bactéries gram négatives (Cani et al., 2007). Les Ips sont produits de façon continue dans I'intestin suite à la lyse des bactéries Gram-négative et sont physiologiquement absorbés puis transportés de l'intestin vers les tissus cibles par un mécanisme dépendant des lipoprotéines. Cette augmentation du taux de Ips a été nommée endotoxémie métabolique et serait impliquée dans les désordres métaboliques associées à l'obésité (insulino-résistance, inflammation systémique, stéatose hépatique...). Au final, des études successives ont permis d'établir qu'un régime hyperlipidique, via une altération du microbiote intestinal, entraîne une augmentation de la perméabilité intestinale et ainsi une endotoxémie métabolique par l'intermédiaire de mécanismes impliquant l'activité endocrine de l'intestin ainsi que le système endocannabinoïde (Delzenne et Cani, 2011).

\section{Métabolisme des lipides par le microbiote intestinal}

Chez I'homme, la quantité de lipides totaux qui parviennent dans le côlon en conditions physiologiques a été évaluée entre 5 et $8 \mathrm{~g}$ par jour, ce chiffre pouvant être considérablement augmenté en situations pathologiques (insuffisance pancréatique, résections intestinales, cholestase. . .) (Juste, 2005). À cela s'ajoutent les lipides bactériens et ceux provenant de la desquamation des colonocytes. Les lipides parvenant dans le côlon subissent de multiples transformations de la part du microbiote intestinal (hydrolyse, oxydation, réduction, hydroxylation...). De nombreuses espèces bactériennes possèdent ainsi des lipases permettant d'hydrolyser les triglycérides à chaînes longues (Juste, 2005). La bio-hydrogénation (ou réduction) des $A G$ insaturés par le microbiote intestinal est quant à elle connue de longue date, révélée par le fait qu'une forte proportion des AG dosés dans les selles sont saturés, cette proportion étant nettement plus faible chez le rat axénique que chez le rat conventionnel. Cependant, tous les AG ne subissent pas ces transformations puisque les AG insaturés à 18 carbones sont réduits par le microbiote intestinal, tandis que les AG à 20 ou 22 carbones ne seraient pas métabolisés. L'hydroxylation de l'acide oléique en acide 10-hydroxy-stéarique constitue un autre exemple du métabolisme des lipides par le microbiote intestinal. Elle consiste en la simple addition d'une molécule d'eau sur la double liaison de l'acide oléique. Enfin, de nombreux micro-organismes, notamment des bactéries Gram-positives, possèdent des activités phospholipasiques. Il existe ainsi des phospholipases et des sphingomyélinases bactériennes différant par la spécificité de leurs substrats et par leurs produits d'hydrolyse. Certains de ces produits, tels les diglycérides et inositol triphosphates, peuvent pénétrer dans les cellules de I'hôte et agir comme messagers intracellulaires, en particulier au sein de voies de signalisation qui contrôlent l'expression de gènes. Il est intéressant de noter que le taux de conversion de la phosphatidylcholine en sn-1,2-DG par des cultures de selles humaines, ainsi que la concentration de $s n-1,2-D G$ dans les selles brutes, sont extrêmement variables d'un individu à l'autre, mais relativement stables dans le temps pour un même individu (Juste, 2005). Cela signifie que pour une même quantité et qualité de lipides parvenant au côlon, les métabolites formés différent d'un individu à l'autre en fonction de la composition de son microbiote intestinal ce qui souligne une fois encore l'influence que peut exercer ce microbiote sur la santé de l'hôte.

Le côlon reçoit également jusqu'à $1 \mathrm{~g}$ par jour de cholestérol dont $70 \%$ provient de la bile, $20 \%$ de la fraction de I'alimentation non absorbée au niveau de l'intestin grêle et les $10 \%$ restants de la desquamation des muqueuses intestinales (Gérard, 2009). Dès les années 1930, il a été montré que le microbiote intestinal était capable de convertir ce cholestérol. Ce métabolisme microbien, pour lequel deux voies (directe et indirecte) ont été proposées, aboutit à la réduction du cholestérol en coprostanol, non absorbé par l'intestin et éliminé dans les fèces (figure 1). Il a ensuite été montré que chez les rats sans germes, l'absorption du cholestérol était augmentée tandis que son excrétion fécale était réduite, aboutissant à une accumulation plus importante de cholestérol dans le foie par rapport à des rats conventionnels. Plusieurs études, réalisées dans les années 1970, ont montré que ce métabolisme du cholestérol suivait une répartition bimodale au sein de la population humaine : chez la majorité des individus, plus de $70 \%$ du cholestérol est ainsi métabolisé par le microbiote, alors que pour une minorité, moins de $20 \%$ du cholestérol est transformé. Récemment, il a été montré que cette répartition était directement liée au nombre de bactéries réductrices de cholestérol présentes dans le tube digestif. Une population minimale de $10^{6} / \mathrm{g}$ de contenu digestif est ainsi nécessaire pour 


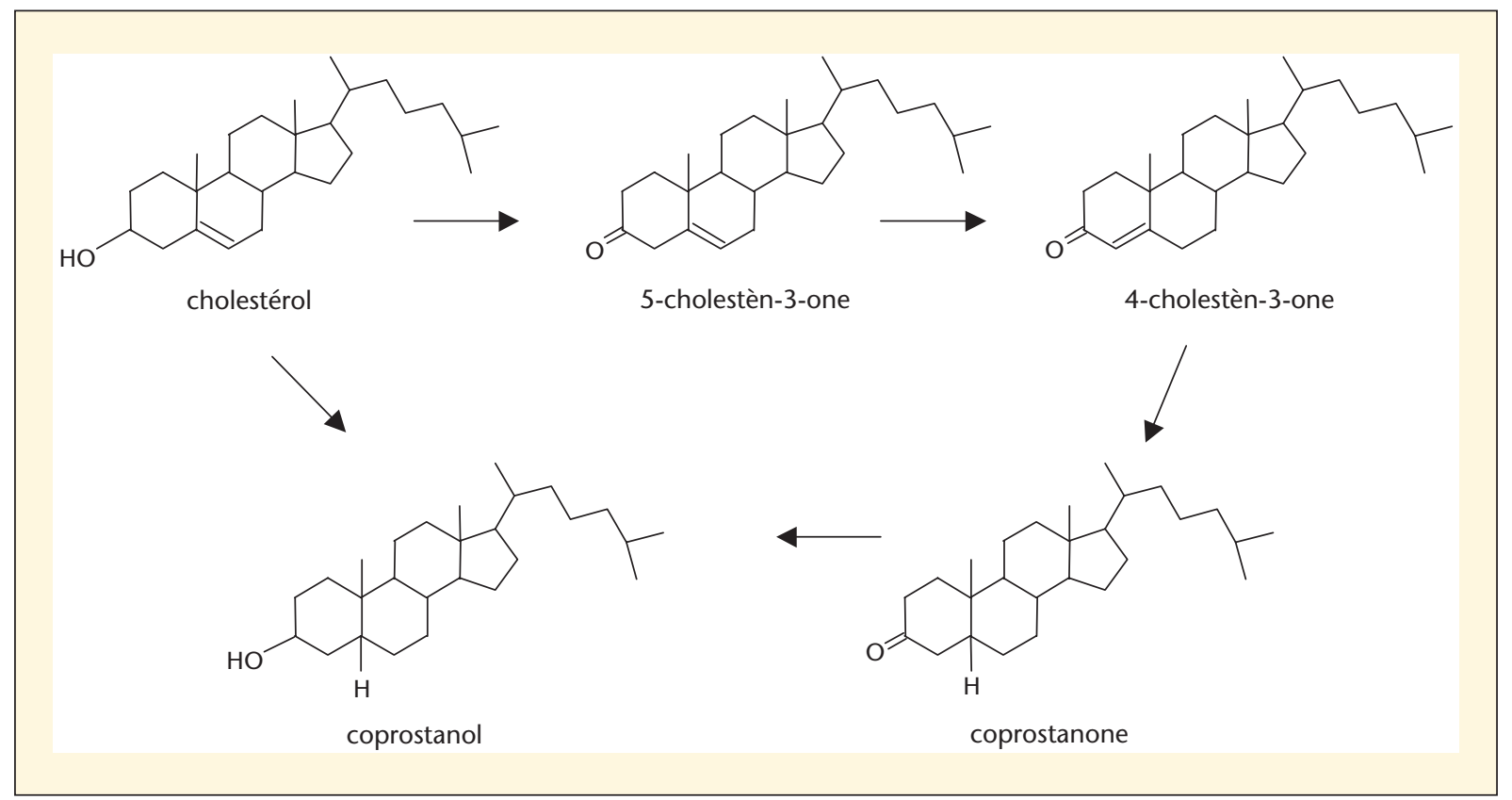

Figure 1. Voies directe et indirecte pour la conversion du cholestérol en coprostanol par le microbiote intestinal.

une conversion partielle du cholestérol tandis qu'une conversion totale nécessite une population de bactéries réductrices de cholestérol supérieure ou égale à $10^{8} / \mathrm{g}$ (Veiga et al., 2005). Si ce métabolisme est donc décrit depuis des décennies, les bactéries responsables restent très mal connues. Ainsi, seules quelques souches actives, toutes classées dans le genre Eubacterium sur des critères phénotypiques, ont d'abord été isolées à partir d'échantillons fécaux de rats et de babouins et, plus récemment, à partir de lisier de porc. Cette dernière souche, un coccobacille anaérobie Gram positif, possédant des propriétés physiologiques et morphologiques uniques, a été définie comme une nouvelle espèce nommée Eubacterium coprostanoligenes ATCC 51222 (Freier et al., 1994). Ce n'est qu'en 2007 qu'une souche bactérienne convertissant le cholestérol et issue d'un microbiote fécal humain a été isolée et caractérisée pour la première fois. Cette souche est étroitement apparentée à l'espèce Bacteroides dorei (Gérard et al., 2007) dont la souche type est cependant incapable de convertir le cholestérol. Au final, si ce métabolisme pourrait limiter l'absorption du cholestérol et donc le risque de maladies cardiovasculaires, l'impact réel du métabolisme microbien intestinal du cholestérol sur la santé humaine n'a jusqu'à présent fait l'objet d'aucune étude.

Les acides biliaires, synthétisés dans le foie à partir du cholestérol, sont ensuite conjugués à la glycine ou à la taurine. Ils sont absorbés au niveau de l'iléon terminal puis transportés par la veine porte au foie où ils sont à nouveau excrétés dans la bile (cycle entérohépatique). Environ $5 \%$ des sels biliaires ( 0,2 à 0,3 g par jour) échappent à ce cycle et parviennent au côlon où ils sont métabolisés par le microbiote en acides biliaires dits secondaires par opposition aux acides biliaires primaires synthétisés par le foie (Gérard, 2009 ; Ridlon et al., 2006). Plus de 20 acides biliaires secondaires ont ainsi été mis en évidence dans les fecès humaines, démontrant la grande variété de conversions possibles des acides biliaires par le microbiote intestinal. Ces transformations incluent la déconjugaison, I'oxydation et l'épimérisation de groupements hydroxyles, la 7-déshydroxylation, la formation de monoesters ou polyesters, ou encore la désulfatation (Ridlon et al., 2006). La déconjugaison est catalysée par une hydrolase spécifique, rencontrée chez des espèces appartenant aux genres Bacteroides, Bifidobacterium, Fusobacterium, Clostridium, Lactobacillus, Listeria et Streptococcus. L'oxydation de groupe- ments hydroxyles est, quant à elle, réalisée par des hydroxystéroïdes déshydrogénases spécifiques des positions C3, C7 ou C12, présentes chez de nombreux genres bactériens, l'action concertée $d^{\prime} \alpha$ et $\beta$-hydroxystéroïdes déshydrogénases aboutissant à l'épimérisation de l'acide biliaire (Ridlon et al., 2006). Chez I'homme, les deux principaux acides biliaires secondaires sont les acides désoxycholique et lithocholique, produits de la $7 \alpha$-déshydroxylation bactérienne des acides biliaires primaires, acides cholique et chénodésoxycholique. Les bactéries intestinales d'origine humaine possédant cette activité ont toutes été identifiées comme appartenant au genre Clostridium (Ridlon et al., 2006). Enfin, des espèces appartenant aux genres Bacteroides ou Citrobacter sont capables de former des polyesters comprenant jusqu'à 22 unités d'acide désoxycholique tandis que des activités sulfatases spécifiques des acides biliaires ont été détectées chez des bactéries du genre Clostridium. II est à noter que ces activités d'hydrolyse des acides biliaires portées par les bactéries intestinales et exprimées pour part dans l'intestin grêle, peuvent réduire l'absorption et augmenter l'excrétion des acides biliaires et, de ce fait, influer sur le niveau de lipides sériques. 


\section{Conclusion}

L'influence du microbiote intestinal sur la santé de l'hôte est maintenant reconnue et fait l'objet d'un nombre grandissant d'études. Récemment, I'implication probable de ce microbiote dans le développement de l'obésité et de pathologies associées a renforcé l'intérêt pour les liens existant entre microbiote intestinal et lipides. Cependant, si plusieurs études ont ainsi démontré que les lipides étaient capables de modifier la composition du microbiote intestinal et qu'en retour des bactéries de ce microbiote métabolisent les lipides, les conséquences en termes de santé de cette interrelation microbiote-lipides restent encore largement à explorer.

\section{RÉFÉRENCES}

Backhed F, Ding $\mathrm{H}$, Wang $\mathrm{T}$, et al. The gut microbiota as an environmental factor that regulates fat storage. Proc Natl Acad Sci U S A $2004 ; 101$ : 15718-23.

Backhed F, Manchester JK, Semenkovich CF, Gordon JI. Mechanisms underlying the resistance to diet-induced obesity in germ-free mice. Proc Natl Acad Sci U S A 2007 ; 104 : 979-84.

Cani PD, Amar J, Iglesias MA, et al. Metabolic endotoxemia initiates obesity and insulin resistance. Diabetes 2007 ; 56 : 1761-72.
De La Cochetiere MF, Durand T, Lepage $P$, et al. Resilience of the dominant human fecal microbiota upon short-course antibiotic challenge. J Clin Microbiol 2005 ; 43 : 5588-92.

Delzenne NM, Cani PD. Interaction between obesity and the gut microbiota : relevance in nutrition. Annu Rev Nutr 2011 ; 31 : 15-31.

Freier TA, Beitz DC, Li L, Hartman PA. Characterization of Eubacterium coprostanoligenes sp. nov., a cholesterol-reducing anaerobe. Int / Syst Bacteriol 1994 ; 44 : 137-42.

Gérard P. Gastrointestinal tract : microbial metabolism of steroids. In : Timmis, Kenneth $\mathrm{N}$ (Ed.), Handbook of Hydrocarbon and Lipid Microbiology. Springer Heidelberg, in press. 2009.

Gérard P, Lepercq $P$, Leclerc $M$, et al. Bacteroides sp. strain D8, the first cholesterol-reducing bacterium isolated from human feces. Appl Environ Microbiol 2007 ; 73 : 5742-9.

Hildebrandt MA, Hoffmann C, Sherrill-Mix $S A$, et al. High-fat diet determines the composition of the murine gut microbiome independently of obesity. Gastroenterology 2009 ; 137 : 1716-24 e1-2.

Juste $C$. Acides gras alimentaires, flore intestinale et cancer. Bull Cancer 2005; 92 : 708-721.

Larsson E, Tremaroli V, Lee YS, et al. Analysis of gut microbial regulation of host gene expression along the length of the gut and regulation of gut microbial ecology through MyD88. Gut 2011 (sous presse); doi :10.1136/gutjnl-2011-301104.
Palmer C, Bik EM, DiGiulio DB, Relman DA, Brown PO. Development of the human infant intestinal microbiota. PLoS Biol 2007 ; 5 : e177.

Rabot S, Membrez M, Bruneau A, et al. Germfree $\mathrm{C} 57 \mathrm{BL} / 6 \mathrm{~J}$ mice are resistant to high-fatdiet-induced insulin resistance and have altered cholesterol metabolism. Faseb J 2010 ; 24 : 4948-59.

Ridlon JM, Kang DJ, Hylemon PB. Bile salt biotransformations by human intestinal bacteria. J Lipid Res 2006 ; 47 : 241-59.

Smith K, McCoy KD, Macpherson AJ. Use of axenic animals in studying the adaptation of mammals to their commensal intestinal microbiota. Semin Immunol 2007 ; 19 : 59-69.

Tap J, Mondot S, Levenez F, et al. Towards the human intestinal microbiota phylogenetic core. Environ Microbiol 2009 ; 11 : 2574-84.

Turnbaugh PJ, Backhed F, Fulton L, Gordon JI. Diet-induced obesity is linked to marked but reversible alterations in the mouse distal gut microbiome. Cell Host Microbe 2008 ; 3 : 21323.

Veiga $\mathrm{P}$, Juste $\mathrm{C}$, Lepercq $\mathrm{P}$, et al. Correlation between faecal microbial community structure and cholesterol-to-coprostanol conversion in the human gut. FEMS Microbiol Lett $2005 ; 242: 81-6$.

Wostmann BS, Larkin C, Moriarty A, Bruckner-Kardoss $E$. Dietary intake, energy metabolism, and excretory losses of adult male germfree Wistar rats. Lab Anim Sci 1983 ; 33 : 46-50. 